\title{
On the Identification of Hammerstein Systems Having Saturation-like Functions with Positive Slopes
}

\author{
Rimantas PUPEIKIS \\ Institute of Mathematics and Informatics \\ Akademijos 4, 08663 Vilnius, Lithuania \\ e-mail:pupeikis@ktl.mii.lt
}

Received: October 2004

\begin{abstract}
The aim of the given paper is the development of an approach for parametric identification of Hammerstein systems with piecewise linear nonlinearities, i.e., when the saturation-like function with unknown slopes is followed by a linear part with unknown parameters. It is shown here that by a simple input data rearrangement and by a following data partition the problem of identification of a nonlinear Hammerstein system could be reduced to the linear parametric estimation problem. Afterwards, estimates of the unknown parameters of linear regression models are calculated by processing respective particles of input-output data. A technique based on ordinary least squares is proposed here for the estimation of parameters of linear and nonlinear parts of the Hammerstein system, including the unknown threshold of the piecewise nonlinearity, too. The results of numerical simulation and identification obtained by processing observations of input-output signals of a discrete-time Hammerstein system with a piecewise nonlinearity with positive slopes by computer are given.
\end{abstract}

Key words: nonlinear systems, system identification, Hammerstein systems, parameter estimation.

\section{Introduction}

A special class of nonlinear systems applied in engineering is Hammerstein systems with hard input nonlinearities. They, usually, consist of a static input nonlinearity and a linear dynamic system that are coupled together. Ordinary examples of hard nonlinearities are the saturation, preload, relay, dead-zone, hysteresis-relay, and hysteresis nonlinearities (Bai, 2002). However, the assumptions that the nonlinearity is invertible or linear in a small region around the origin are not satisfied for most hard nonlinearities, because they cannot be described by polynomials and are noninvertible in general. On the other hand, the Hammerstein systems are common in nonlinear control applications (Bai, 2002; Billings and Fakhouri, 1979; Glad and Ljung, 2000; Guo and Bretthauer, 2003; Haist et al., 1973; Hasiewisz and Mzyk, 2004; Hunter and Korenberg, 1986; Janczak, 1999; Ljung, 1999; Narendra and Gallman, 1966; Pawlak, 1991; Sjöberg et al., 1995; Vörös, 1995; Vörös, 2003). Frequently as an input nonlinearity the piecewise saturation-like nonlinearity is used here, too. Assuming the nonlinearity to be piece- 
wise linear, one could let the nonlinear part of the Hammerstein system be represented by different regression functions with some parameters, that are unknown beforehand. In such a case, observations of the input of a Hammerstein system could be partitioned into distinct data sets according to different descriptions. The boundaries of sets of observations depend on the value of the unknown threshold $a$-observations are divided into regimes subject to whether the some observed threshold variable is smaller or larger than $a$ (Hansen and Seo, 2002; Roll, 2003). Therefore the problem of the identification of unknown parameters of nonlinear and linear blocks of the Hammerstein systems could be solved, if a simple way of partitioning the available data sets were found in the case of unknown $a$. Afterwards, the estimates of parameters of regression functions could be calculated by processing particles of observations to be determined (Pupeikis, 2005).

The next section introduces the statement of the problem to be solved. In Section 3, we solve the problem using the data rearrangement by the following reconstruction of the unknown intermediate signal. In Section 4, simulation results are presented. Section 5 contains conclusions.

\section{Statement of the Problem}

The Wiener and Hammerstein systems are subdivided into linear dynamic and nonlinear static blocks. The Wiener system consists of a linear dynamic part followed by a static nonlinearity (Fig. 1). The Hammerstein system (Fig. 2) could be treated as the structurally reversed Wiener system. It consists of a static nonlinearity $f(\cdot, \eta)$ followed by a linear part $G\left(q^{-1}, \boldsymbol{\Theta}\right)$. The linear part of the Hammerstein system is dynamic, time invariant, causal, and stable. It can be represented by a time invariant dynamic system (LTI) with the transfer function $G\left(q^{-1}, \Theta\right)$ as a rational invertible function of the form

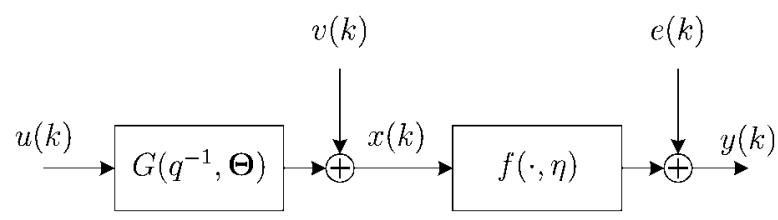

Fig. 1. The Wiener system with the process noise $v(k)$ and that of the measurement $e(k)$. The linear dynamic part $G\left(q^{-1}, \boldsymbol{\Theta}\right)$ of the Wiener system is parametrised by $\boldsymbol{\Theta}$, while the static nonlinear part $f(\cdot, \eta)-$ by $\eta$. Signals: $u(k)$ is input, $y(k)$ is output, $x(k)$ is an unmeasurable intermediate signal.

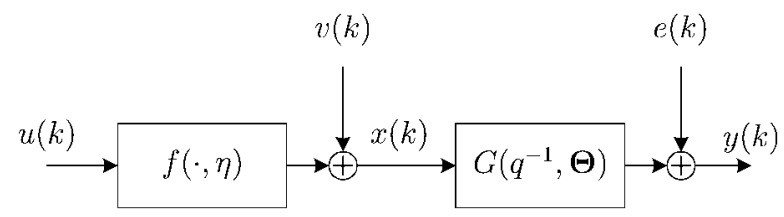

Fig. 2. The Hammerstein system with the process noise $v(k)$ and that of the measurement $e(k)$. The values and notation are the same as in (Fig. 1). 


$$
G\left(q^{-1}, \boldsymbol{\Theta}\right)=\frac{b_{0}+b_{1} q^{-1}+\ldots+b_{m} q^{-m}}{1+a_{1} q^{-1}+\ldots+a_{m} q^{-m}}=\frac{B\left(q^{-1}, \mathbf{b}\right)}{1+A\left(q^{-1}, \mathbf{a}\right)}
$$

with a finite number of parameters

$$
\boldsymbol{\Theta}^{T}=\left(b_{0}, b_{1}, \ldots, b_{m}, a_{1}, \ldots, a_{m}\right), \mathbf{b}^{T}=\left(b_{0}, b_{1}, \ldots, b_{m}\right), \mathbf{a}^{T}=\left(a_{1}, \ldots, a_{m}\right),
$$

that are determined from the set $\boldsymbol{\Omega}$ of permissible parameter values $\boldsymbol{\Theta}$. Here $q^{-1}$ is a backward time-shift operator; the set $\Omega$ is restricted by conditions on the stability of the respective difference equation. The output signal

$$
y(k)=\frac{B\left(q^{-1}, \mathbf{b}\right)}{1+A\left(q^{-1}, \mathbf{a}\right)} x(k)+e(k)
$$

is generated by the linear part of the Hammerstein system (1) as a response to the unknown intermediate signal

$$
x(k)=f(u(k), \eta)+v(k) .
$$

Here the nonlinear part $f(\cdot, \eta)$ with the vector of parameters $\eta$ is a saturation-like function of the form (Roll, 2003)

$$
f(u(k), \eta)= \begin{cases}c_{0}+c_{1} u(k) & \text { if } u(k) \leqslant-a, \\ u(k) & \text { if }-a<u(k) \leqslant a, \\ d_{0}+d_{1} u(k) & \text { if } u(k)>a\end{cases}
$$

that could be partitioned into three functions. These functions are:

$f\{u(k ; \boldsymbol{\Theta}), \mathbf{c}, a\}=c_{0}+c_{1} u(k), f\{u(k ; \boldsymbol{\Theta}), a\}=u(k)$, and $f\{u(k ; \boldsymbol{\Theta}), \mathbf{d}, a\}=$ $d_{0}+d_{1} u(k)$. The function $f\{u(k ; \boldsymbol{\Theta}), \mathbf{c}, a\}$ has only negative values, when $u(k) \leqslant-a$, $f\{u(k ; \boldsymbol{\Theta}), a\}$ has arbitrary positive, as well as negative values, when $-a<u(k) \leqslant a$, and $f\{u(k ; \boldsymbol{\Theta}), \mathbf{d}, a\}$ has only positive values, when $u(k)>a$. Here $u(k ; \boldsymbol{\Theta}) \equiv$ $u(k), \mathbf{c}^{T}=\left(c_{0}, c_{1}\right), c_{0}=-a\left(1-c_{1}\right), 0<c_{1}<a, \mathbf{d}^{T}=\left(d_{0}, d_{1}\right), d_{0}=a\left(1-d_{1}\right), 0<$ $d_{1}<a$.

The process noise $v(k) \equiv \xi(k)$ and the measurement noise $e(k) \equiv \zeta(k)$ are added to an intermediate signal $x(k)$ and the output $y(k)$, respectively, $\xi(k), \zeta(k)$ are mutually noncorrelated sequences of independent Gaussian variables with $E\{\xi(k)\}=$ $0, E\{\zeta(k)\}=0, E\{\xi(k) \xi(k+\tau)\}=\sigma_{\xi}^{2} \delta(\tau), E\{\zeta(k) \zeta(k+\tau)\}=\sigma_{\zeta}^{2} \delta(\tau) ; E\{\cdot\}$ is a mean value, $\sigma_{\zeta}^{2}, \sigma_{\xi}^{2}$ are variances of $\zeta$ and $\xi$, respectively, $\delta(\tau)$ is the Kronecker delta function.

The aim of the given paper is to estimate parameters (2) of the linear part (1), as well as parameters $\eta=\left(c_{0}, c_{1}, d_{0}, d_{1}\right)^{T}$ and the threshold $a$ of nonlinearity (5) by processing $N$ pairs of observations $u(k)$ and $y(k)$ of the Hammerstein system (Fig. 2). 


\section{The Data Reordering}

Let us rearrange the data $u(k) \forall k \in \overline{1, N}$ in an ascending order of their values. Thus, the observations of the rearranged input $\tilde{u}(k)$ of the Hammerstein system should be partitioned into three data sets: left-hand side data set ( $N_{1}$ samples) with values lower than or equal to negative $a$, middle data set ( $N_{2}$ samples) with values higher than negative $a$ but lower or equal to $a$, and right-hand side data set ( $N_{3}$ samples) with values higher than $a$. Here $N=N_{1}+N_{2}+N_{3}$. From the engineering point of view it is assumed that no less than $50 \%$ observations are concentrated on the middle-set and approximately by $25 \%$ or less on any side set. Hence, the observations of the rearranged input $\tilde{u}(k)$ with the highest and positive values will be concentrated on the right-hand side set, while the observations with the lowest and negative values on the left-hand side one. Thus, the middle data set $\tilde{u}(k) \forall k \in \overline{N_{1}+1, N_{2}}$ is, really, reordered in an ascending order of their values $u(k) \forall k \in \overline{1, N}$ with some portions of missing observations within it that belong to the left-hand and right-hand side sets of the data.

Let us suppose now that the process noise $v(k)$ is absent. Then the observations of the unknown intermediate signal $x(k)$ are coincident with the respective observations of the middle data set $\tilde{u}(k)$ equivalent to those input observations $u(k)$ that passed the piecewise nonlinearity (5) without any processing. In such a case, one could get these observations simply by choosing the upper interval bound lower than the 75 percentage and the lower interval bound higher than the 25 percentage of the sampled reordered observations of $\tilde{u}(k)$.

Let us now partially reconstruct an unmeasurable intermediate signal $x(k)$, choosing in the initial order only those values of $u(k) \forall k \in \overline{1, N}$ that are present in the middle data set of $\tilde{u}(k) \forall k \in \overline{N_{1}+l_{1}, N_{2}-l_{2}}$. Here $l_{1}, l_{2}$ are some portions of observations of the middle data set of $\tilde{u}(k) \forall k \in \overline{N_{1}+1, N_{2}}$. In such a case one could get $x(k) \equiv u(k)$ for $k=1+l(k)$, such that $l(k) \leqslant l(k+1)$, where $l(k)$ is a positive time-varying integer. Really, assuming that the process noise $v(k)$ is absent, the available sequence $x(k)$ is equivalent to the input sequence $u(k)$ but with some portions of missing observations in it that belong to the left-hand or right-hand side sets of the rearranged data. It could be used to calculate the estimates of parameters (2) of the transfer function $G(q, \boldsymbol{\Theta})$ according to

$$
\widehat{\boldsymbol{\Theta}}=\left(\widetilde{\mathbf{X}}^{T} \widetilde{\mathbf{X}}\right)^{-1} \widetilde{\mathbf{X}}^{T} \widetilde{\mathbf{Y}}
$$

if a number of equations of the initial system of linear equations

$$
\mathbf{Y}=\mathbf{X} \Theta
$$

with

$$
\mathbf{X}=\left[\begin{array}{cccccc}
x(m+\nu) & \ldots & x(\nu) & -y(m+\nu) & \ldots & -y(\nu) \\
x(m+\nu+1) & \ldots & x(\nu+1) & -y(m+\nu+1) & \ldots & -y(\nu+1) \\
\vdots & & \vdots & \vdots & & \vdots \\
x(N-1) & \ldots & x(N-m) & -y(N-1) & \ldots & -y(N-m)
\end{array}\right]
$$


and $\mathbf{Y}=(y(m+\nu+1), y(m+\nu+2), \ldots, y(N))^{T}$, that correspond to the left- and right-hand side sets of the reordered input data are rejected beforehand. Here

$$
\widehat{\boldsymbol{\Theta}}^{T}=(\hat{\mathbf{b}}, \hat{\mathbf{a}})^{T}, \quad \hat{\mathbf{b}}^{T}=\left(\hat{b}_{0}, \hat{b}_{1}, \ldots, \hat{b}_{m}\right), \quad \hat{\mathbf{a}}^{T}=\left(\hat{a}_{1}, \ldots, \hat{a}_{m}\right)
$$

are $(2 m+1) \times 1,(m+1) \times 1, m \times 1$ vectors of the estimates of parameters (2), respectively, $\widetilde{\mathbf{X}}$ is the $(L-m) \times(2 m+1)$ matrix, and $\widetilde{\mathbf{Y}}$ is the $(L-m-1) \times 1$ vector that are obtained by rejecting respective number of rows of the system (7), and $L=N-N_{1}-N_{3}-l_{1}-l_{2}$ is the whole number of observations of the middle data set $\tilde{u}(k) \forall k \in \overline{N_{1}+l_{1}, N_{2}-l_{2}}$ to be processed.

Afterwards, one could completely reconstruct the unknown intermediate signal $x(k) \forall k \in \overline{1, N}$ according to the formula

$$
\hat{x}(k)=G^{-1}\left(q^{-1}, \widehat{\boldsymbol{\Theta}}\right) y(k)=\frac{1+A\left(q^{-1}, \hat{\mathbf{a}}\right)}{B\left(q^{-1}, \hat{\mathbf{b}}\right)} y(k)
$$

or the expression in an extended form

$$
\begin{aligned}
\hat{x}(k)= & \frac{1}{\hat{b}_{0}} y(k)+\frac{\hat{a}_{1}}{\hat{b}_{0}} y(k-1)+\ldots+\frac{\hat{a}_{m}}{\hat{b}_{0}} y(k-m) \\
& -\frac{\hat{b}_{1}}{\hat{b}_{0}} \hat{x}(k-1)-\ldots-\frac{\hat{b}_{m}}{\hat{b}_{0}} \hat{x}(k-m),
\end{aligned}
$$

if in (1) their estimates are substituted instead of respective parameter sets (2). Here $\hat{x}(k)$ is an estimate of $x(k)$ consisting of their reconstructed values, $\hat{b}_{0} \neq 0$.

Estimates of the parameters $c_{0}, d_{0}$ and $c_{1}, d_{1}$ are calculated by the ordinary least squares, too. In such a case, the sums of the form

$$
\begin{aligned}
& I\left(c_{0}, c_{1}\right)=\sum_{i=1}^{N_{1}}\left[\tilde{\hat{x}}(i)-c_{0}-c_{1} \tilde{u}(i)\right]^{2}=\min ! \\
& I\left(d_{0}, d_{1}\right)=\sum_{j=N_{1}+L+1}^{N}\left[\tilde{\hat{x}}(j)-d_{0}-d_{1} \tilde{u}(j)\right]^{2}=\min !
\end{aligned}
$$

are to be minimized in respect of parameters $c_{0}, c_{1}$ and $d_{0}, d_{1}$, respectively, using sideset data particles of $\hat{x}(k)$ and respective observations of the rearranged input signal $\tilde{u}(k)$. Here $\tilde{\hat{x}}(k)$ are observations of the signal $\hat{x}(k)$ that were rearranged in accordance with $\tilde{u}(k)$.

The estimates of parameters $c_{1}, d_{1}$ and $c_{0}, d_{0}$ are calculated according to (Malinvaud, 1969)

$$
\begin{array}{ll}
\hat{c}_{1}=\frac{\sum_{i=1}^{N_{1}} \tilde{\hat{x}}(i) \tilde{u}(i)}{\sum_{i=1}^{N_{1}} \tilde{u}^{2}(i)}, & \hat{d}_{1}=\frac{\sum_{j=1}^{N_{3}} \tilde{\hat{x}}(j) \tilde{u}(j)}{\sum_{j=1}^{N_{3}} \tilde{u}^{2}(j)}, \\
\hat{c}_{0}=\frac{\sum_{i=1}^{N_{1}}\left[\tilde{\hat{x}}(i)-\hat{c}_{1} \tilde{u}(i)\right]}{N_{1}}, & \hat{d}_{0}=\frac{\sum_{j=1}^{N_{3}}\left[\tilde{\hat{x}}(j)-\hat{d}_{1} \tilde{u}(j)\right]}{N_{3}},
\end{array}
$$


respectively, but using side-sets data particles of $\tilde{u}(k)$ and associated observations of the auxiliary signal $\hat{x}(k)$, that are reordered in accordance with $\tilde{u}(k)$.

The estimates of the threshold $a$ for the right-hand side and left-hand side sets are found according to

$$
\hat{a}=\hat{d}_{0} /\left(1-\hat{d}_{1}\right), \quad \hat{a}=\hat{c}_{0} /\left(1-\hat{c}_{1}\right),
$$

respectively.

It should be noted that the simple input and associated output data reordering with a following reconstruction of an intermediate signal that is really unknown, allow us to turn the nonlinear problem of parametric estimation of Hammerstein systems to a linear one where linear estimators based on the ordinary LS, are efficient. The presented algorithm is not only adapted to the specific nonlinearity considered with a limited general interest, - the procedure used in data reordering could be applied to robust parametric identification of LTI dynamic systems, by processing output and noisy input observations in the presence of lonely or patchy outliers of large magnitude. The same approach, based on the data reordering but used for parametric identification of the Wiener system (Fig. 1) with the nonlinearity of the form (5), is analysed in (Pupeikis, 2003). In such a case, all observations of the input are available for processing. Therefore the FIR (finite impulse response) model could be used to replace the initial transfer function $G\left(q^{-1}, \boldsymbol{\Theta}\right)$ of the linear part of the Wiener system. Thus, the dependence of some regressors on the process output will be diminished, and the assumption that the regressors depend only on the non-noisy input signal will be satisfied. Besides, by applying the FIR model for the parametric identification of the Wiener system one avoids the influence of some missing regressors, appearing in the regression matrix. This opportunity does not take place in the Hammerstein system (Fig. 2) due to the presence of some missing observations in the input sequence.

\section{Numerical Simulation}

The true non-noisy intermediate signal $x(k) k=\overline{1, N}$, of the Hammerstein system (Figs. 3b, 4b) is given by

$$
x(k)= \begin{cases}-0.9+0.1 u(k) & \text { if } u(k) \leqslant-1, \\ u(k) & \text { if }-1<u(k) \leqslant 1, \\ 0.9+0.1 u(k) & \text { if } u(k)>1\end{cases}
$$

with the sum of sinusoids (Fig. 3a)

$$
u(k)=\frac{1}{20} \sum_{i=1}^{20} \sin \left(i \pi k / 10+\phi_{i}\right)
$$



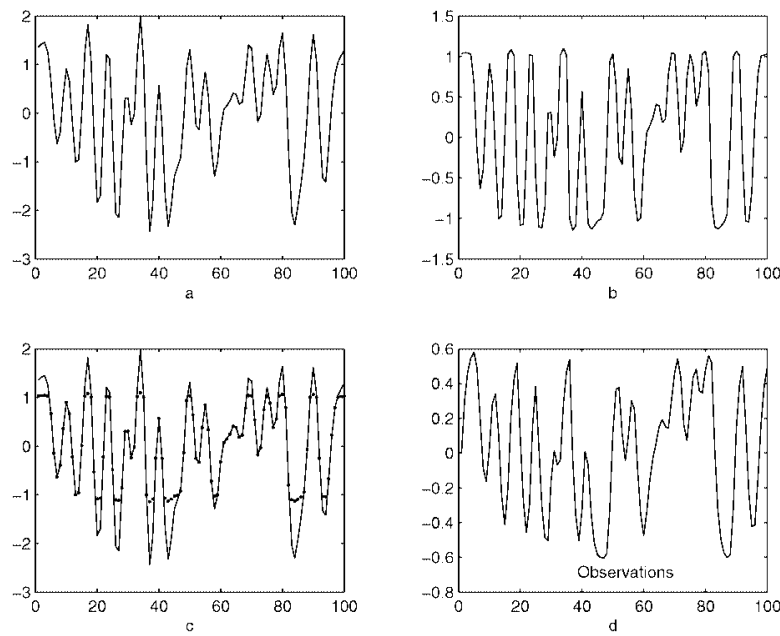

Fig. 3. The signals of the simulated Hammerstein system with a piecewise nonlinearity (17): input $u(k)$, calculated by (18)(a), intermediate signal $x(k)$ (b), input $u(k)$ and intermediate signal $x(k)$ (dotted line)(c) and output $y(k)(\mathrm{d})$
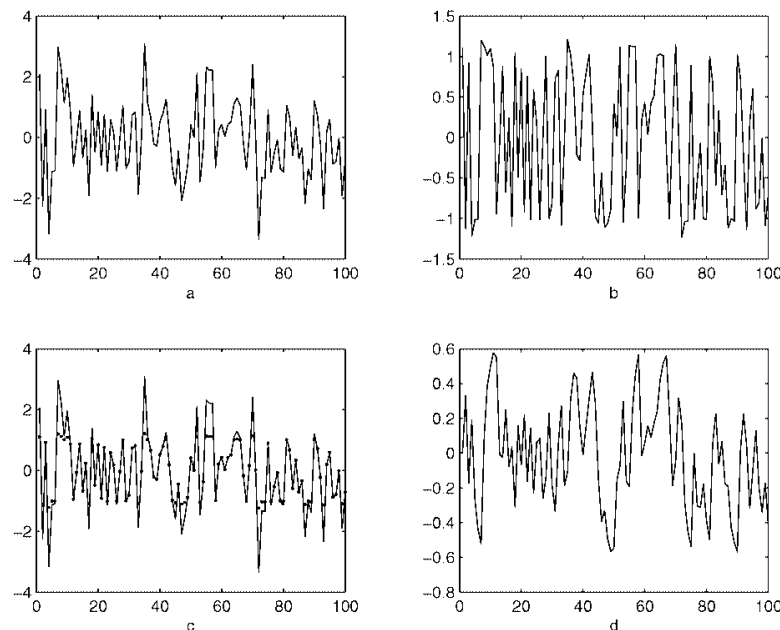

Fig. 4. The signals of the simulated Hammerstein system with a piecewise nonlinearity (17): input $u(k)$ is white Gaussian noise (a), intermediate signal $x(k)$ (b), input $u(k)$ and intermediate signal $x(k)$ (dotted line)(c) and output $y(k)(\mathrm{d})$

and white Gaussian noise with variance 1.7 (Fig. 4a) as inputs to the nonlinear block. Here in (18) the stochastic variables $\phi_{k}$ with a uniform distribution on $[0,2 \pi]$ were chosen. The true output signal (Figs. 3d, 4d) is described by

$$
y(k)=G\left(q^{-1}, \boldsymbol{\Theta}\right) x(k)=\frac{b_{1} q^{-1}}{1+a_{1} q^{-1}} x(k)
$$

with $b_{1}=0.3$ and $a_{1}=-0.5$. 

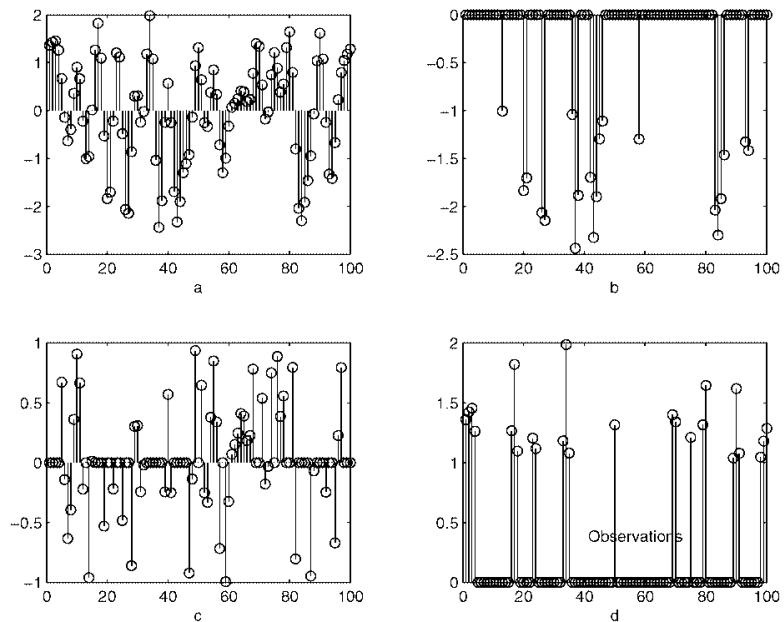

Fig. 5. Samples of $u(k)$ (a) (see Fig. 3a) and its data sets: left (b), middle (c), right (d)(here the observations, that belong to the other data set, are equal to zeros). Input $u(k)$ of the form (18).
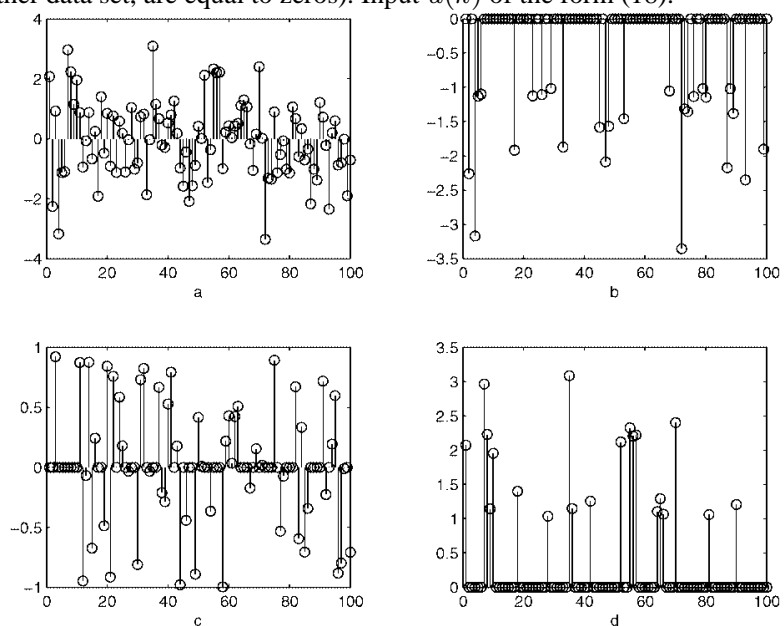

Fig. 6. Samples of signal $u(k)$ (a) (see Fig. 4a) and its data sets: left (b), middle (c), right (d). Input $u(k)$ is white Gaussian noise.

First of all, $N=100$ data points have been generated without additive process and measurement noises (Figs. 3, 4). Afterwards, the LS problem (6) was solved, using 56 and 55 rearranged observations of the input, respectively (Figs. 5c, 6c), excluding zeros, and associated observations of the respective outputs (Figs. 3d, 4d). The estimate $\hat{x}(k)$ of the intermediate signal $x(k)$ was reconstructed according to

$$
\hat{x}(k)=\frac{1}{\hat{b}_{1}} y(k+1)+\frac{\hat{a}_{1}}{\hat{b}_{1}} y(k),
$$



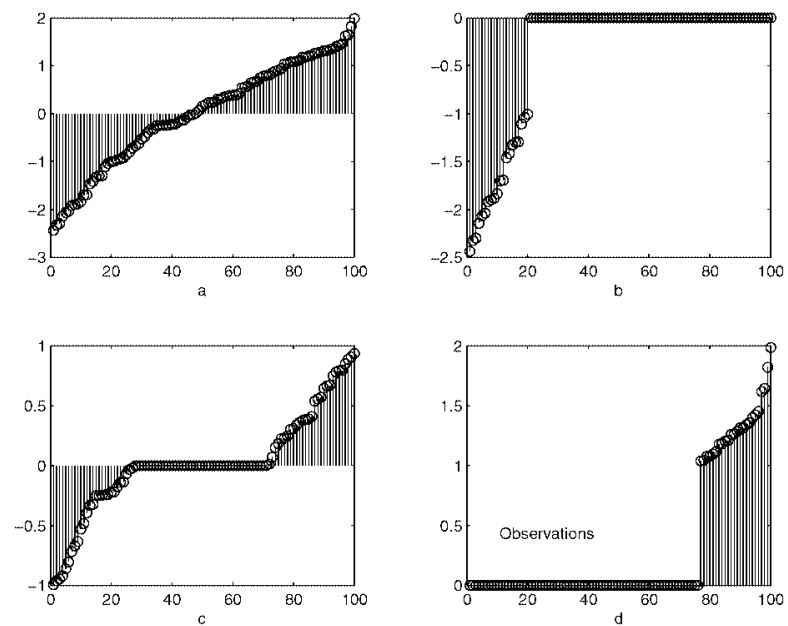

Fig. 7. The reordered in an ascending order of their values signal $u(k)$ (a) (see Fig. 5a) and its rearranged data sets: left (b), middle (c), right (d) (here the observations, that belong to the other data set, are equal to zeros).
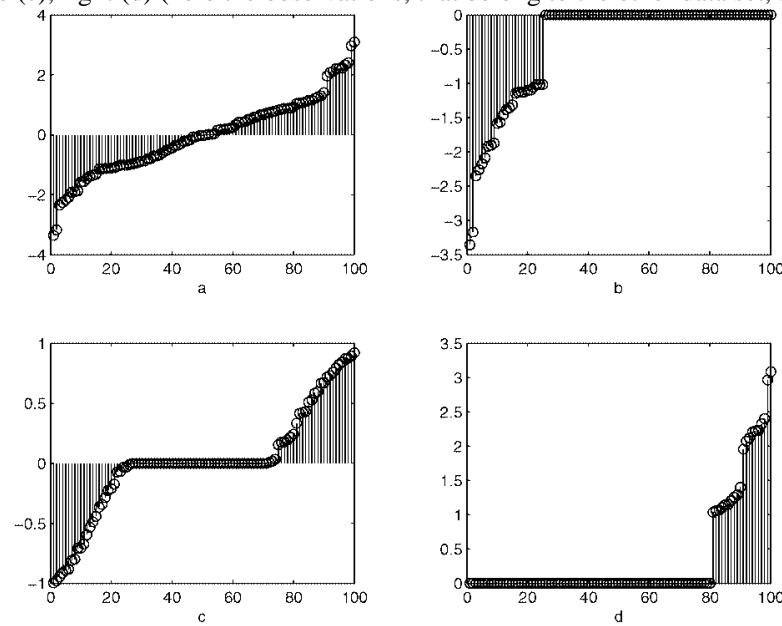

Fig. 8. The reordered in an ascending order of their values signal $u(k)$ (a) (see Fig. 6a) and its rearranged data sets: left (b), middle (c), right (d).

replacing unknown true values of parameters by their estimates. In such a case, the estimates $\hat{b}_{1}, \hat{a}_{1}$ were approximately equal to the true parameters: $b_{1}=0.3, a_{1}=0.5$. The reconstructed versions of the intermediate signal $x(k)$ are shown in (Figs. 9a, b).

It ought to be noted that the accuracy of estimates of the intermediate signal, calculated by formula (20), for both inputs is the same, when the process- and measurement noises are absent. If $\hat{x}(k)$ has been obtained, then it is simple to separate different particles of observations of $\hat{x}(k)$ that belong to the respective side-sets of reordered $u(k)$ (Figs. 5-8). The estimates of parameters $c_{1}, d_{1}$ and $c_{0}, d_{0}$ are calculated according to formulas (14) and (15), respectively. In such a case, the rearranged observations of $\hat{x}(k)$ and $u(k)$ were substituted in formulas (14) and the estimates of $c_{1}$ and $d_{1}$ were deter- 

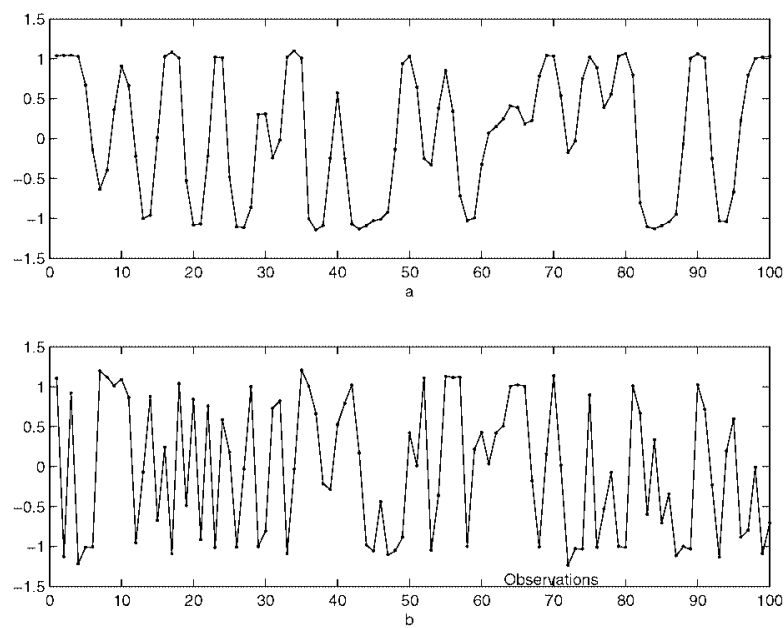

Fig. 9. The intermediate signal $x(k)$ (continuous line), and the reconstructed versions of $x(k)$ (dotted line), calculated using Eq. (11) with the sum of sinusoids (Fig. 3a) (a) and white Gaussian noise (Fig. 4a) (b).

mined: $\hat{c}_{1}=\hat{d}_{1}=0.1$. Then, the estimates $\hat{c}_{0}$ and $\hat{d}_{0}$ were calculated by (15). Their values are also coincidental with the values of true coefficients: $\hat{c}_{0}=-0.9$, while $\hat{d}_{0}=0.9$. It should be noted that $N_{1}=19, N_{3}=23$ for the periodical signal (18) (Fig. 3a) and $N_{1}=25, N_{3}=20$ for the Gaussian white noise (Fig. 4a) were used to calculate the estimates $\hat{c}_{0}, \hat{c}_{1}, \hat{d}_{0}, \hat{d}_{1}$, respectively. The estimates of the threshold were established by Eqs. (16). The values of estimates $\hat{a}$ were equal to the true value $a=1$.

In order to determine how realizations of different process- and measurement noises affect the accuracy of estimation of unknown parameters, we have used the Monte Carlo simulation with 10 data samples, each containing 100 pairs of input-output observations. 10 experiments with the same realization of the process noise $v(k)$ and different realizations of the measurement noise $e(k)$ with different levels of its intensity have been carried out. The intensity of noises was assured by choosing respective signal-to-noise ratios SNR (the square root of the ratio of signal and noise variances). For the process noise, $\mathrm{SNR}^{v}$ was equal to 100 , and for the measurement noise, $\mathrm{SNR}^{e}: 1,10,100$. As inputs for all given nonlinearities the periodical signal (18) and white Gaussian noise were chosen. In each $i$ th experiment the estimates of parameters were calculated. During the Monte Carlo simulation averaged values of estimates of the parameters and of the threshold and their confidence intervals were calculated. In Tables 1 and 2, for each input the averaged estimates of parameters and the threshold $a$ of the simulated Hammerstein system (Fig. 2) with the linear part (19) $\left(b_{1}=0.3 ; a_{1}=-0.5\right)$ and the piecewise nonlinearity (17) $\left(c_{0}=-0.9, c_{1}=0.1, d_{0}=0.9, d_{1}=0.1\right)$ with their confidence intervals are presented. It ought to be noted that in each experiment here the value of $\mathrm{SNR}^{v}$ was fixed and was the same, while the values of $\mathrm{SNR}^{e}$ were varying due to different realizations of $e(k)$. The Monte Carlo simulation (Tables 1,2) implies that the accuracy of parametric identification of the Hammerstein system depends on the intensity of measurement noise. 
Table 1

Averaged estimates of the parameters $b_{1}, a_{1}, c_{0}, c_{1}, d_{0}, d_{1}$, and thresholds $a,-a$ with their confidence intervals. Input: the periodical signal. $\mathrm{SNR}^{v}=100$

\begin{tabular}{crrr}
\hline Estimates & $\mathrm{SNR}^{e}=1$ & $\mathrm{SNR}^{e}=10$ & $\mathrm{SNR}^{e}=100$ \\
\hline$\hat{b}_{1}$ & $0.39 \pm 0.10$ & $0.32 \pm 0.03$ & $0.3 \pm 0.01$ \\
$\hat{a}_{1}$ & $-0.09 \pm 0.09$ & $-0.39 \pm 0.04$ & $-0.48 \pm 0.01$ \\
\hline$\hat{c}_{0}$ & $-1.20 \pm 0.39$ & $-1.04 \pm 0.09$ & $-0.99 \pm 0.03$ \\
$\hat{c}_{1}$ & $0.02 \pm 0.24$ & $0.04 \pm 0.07$ & $0.06 \pm 0.02$ \\
\hline$\hat{d}_{0}$ & $0.19 \pm 0.83$ & $0.55 \pm 0.30$ & $0.78 \pm 0.1$ \\
$\hat{d}_{1}$ & $0.57 \pm 0.61$ & $0.31 \pm 0.21$ & $0.15 \pm 0.07$ \\
\hline$\hat{a}$ & $0.62 \pm 0.83$ & $0.57 \pm 0.55$ & $0.91 \pm 0.06$ \\
\hline$-\hat{a}$ & $-1.23 \pm 0.36$ & $-1.09 \pm 0.08$ & $-1.05 \pm 0.02$ \\
\hline
\end{tabular}

Table 2

The values and notation are the same as in Table 1. Input - the Gaussian white noise

\begin{tabular}{crrr}
\hline Estimates & $\mathrm{SNR}^{e}=1$ & $\mathrm{SNR}^{e}=10$ & $\mathrm{SNR}^{e}=100$ \\
\hline$\hat{b}_{1}$ & $0.34 \pm 0.05$ & $0.32 \pm 0.01$ & $0.3 \pm 0.00$ \\
$\hat{a}_{1}$ & $-0.17 \pm 0.11$ & $-0.42 \pm 0.04$ & $-0.48 \pm 0.02$ \\
\hline$\hat{c}_{0}$ & $-1.08 \pm 0.51$ & $-0.95 \pm 0.16$ & $-0.93 \pm 0.05$ \\
$\hat{c}_{1}$ & $0.02 \pm 0.28$ & $0.07 \pm 0.08$ & $0.07 \pm 0.03$ \\
\hline$\hat{d}_{0}$ & $0.94 \pm 0.34$ & $0.91 \pm 0.13$ & $0.89 \pm 0.04$ \\
$\hat{d}_{1}$ & $0.07 \pm 0.18$ & $0.08 \pm 0.07$ & $0.09 \pm 0.02$ \\
\hline$\hat{a}$ & $0.97 \pm 0.22$ & $0.98 \pm 0.08$ & $0.98 \pm 0.03$ \\
\hline$-\hat{a}$ & $-1.05 \pm 0.25$ & $-1 \pm 0.09$ & $-1 \pm 0.03$ \\
\hline
\end{tabular}

In the absence of process and measurement noises, estimates of the parameters and thresholds, obtained for the Hammerstein as well as Wiener systems having identical linear and nonlinear parts, are the same as the respective true values of parameters and thresholds, if the assumption that no less than $50 \%$ observations are concentrated on the middle-set and approximately by $25 \%$ or less on any side set is valid. On the other hand, in the presence of abovementioned noises, estimates of parameters of both systems are different due to artificial correlating of process noise $v(k)$ by the linear part of the Hammerstein system. In such a case, the estimates for the Wiener system are more accurate than those derived for the Hammerstein one, especially, for intensive noises (Pupeikis, 2003). 


\section{Conclusions}

A problem of identification of Hammerstein systems having saturation-like functions could be essentially reduced by a simple data rearrangement in an ascending order according to their values. Thus, the available input data are partitioned into three data sets that correspond to distinct threshold regression models. Later on the estimates of unknown parameters of linear regression models can be calculated by processing respective sets of the rearranged input and associated output observations. A technique, based on ordinary LS, is proposed here for estimating the parameters of linear and nonlinear parts of the Hammerstein system (Fig. 2), including the unknown threshold of the piecewise nonlinearity, too. During successive steps the unknown intermediate signal is reconstructed and the missing values of observations of respective data particles are replaced by their estimates. Various results of numerical simulation (Figs. 3-9), including that of Monte Carlo (Tables 1,2) prove the efficiency of the proposed approach for the parametric identification of Hammerstein systems.

\section{References}

Bai, E.-W. (2002). Identification of linear systems with hard input nonlinearities of known structure. Automatica, 38, 853-860.

Billings, S.A., and S.Y. Fakhouri (1979). Nonlinear system identification using the Hammerstein model. International Journal of System Sciences, 10, 567-578.

Glad, T., and L. Ljung (2000). Control Theory, Multivariable and Nonlinear Methods. Taylor \& Francis.

Guo, F., and G. Bretthauer (2003). Identification of generalized Wiener and Hammerstein systems. In IJCI Proceedings of International Conference on Signal Processing, vol. 1(2). pp. 544-578.

Haist, N.D., F.H.I. Chang, and R. Luus (1973). Nonlinear identification in the presence of correlated noise using a Hammerstein model. IEEE Transactions on Automatic Control, 552-555.

Hansen, B.E., and B. Seo (2002). Testing for two-regime threshold cointegration in vector error-correction models. Journ. of Econometrics, 110, 293-318.

Hasiewisz, Z., and G. Mzyk (2004). Combined parametric-nonparametric identification of Hammerstein systems. IEEE Transactions on Automatic Control, 49(8), 1370-1375.

Hunter, I.W., and M.J. Korenberg (1986). The identification of nonlinear biological systems: Wiener and Hammerstein cascade models. Biolog. Cybern., 55, 135-144.

Janczak, A. (1999). Parameter estimation based fault detection and isolation in Wiener and Hammerstein systems. Int. J. Appl. Math. and Comp. Sci., 9(3), 741-735.

Ljung, L. (1999). System Identification, Theory for the User. Second edition. Prentice-Hall PTR.

Malinvaud, E. (1969). Statistical Methods of Econometrics. North-Holland, Amsterdam.

Narendra, K.S., and P.G. Gallman (1966). An iterative method for the identification of nonlinear systems using a Hammerstein model. IEEE Transactions on Automatic Control, 546-550.

Pawlak, M. (1991). On the series expansion approach to the identification of Hammerstein systems. IEEE Transactions on Automatic Control, 36, 763-767.

Pupeikis, R. (2003). On the identification of Wiener systems. Liet. matem. rink., 43, spec. issue, 643-648.

Pupeikis, R. (2005). On the identification of Wiener systems having saturation-like functions with positive slopes. Informatica, 16(1), 131-144.

Roll, J. (2003). Local and piecewise affine approaches to system identification. PhD thesis No 802. Depart. of Electr. Engineer., Linköping University, Linköping, Sweden, March 2003.

Sjöberg, J., Q. Zhang, L. Ljung, A. Benveniste, B. Delyon, P.Y. Glorennec, H. Hjalmarsson, and A. Juditsky (1995). Nonlinear black-box modeling in system identification: a unified overview. Automatica, 31(12), 1691-1724. 
Vörös, J. (1995). Identification of nonlinear dynamic systems using extended Hammerstein and Wiener models. Control Theory and Advanced Technology, 10(4), part 2, 1203-1212.

Vörös, J. (2003). Recursive identification of Hammerstein systems with discontinuous nonlinearities containing dead-zones. IEEE Transactions on Automatic Control, 48(12), 2203-2206.

R. Pupeikis received $\mathrm{PhD}$ degree from Kaunas Polytechnic Institute, Kaunas, Lithuania, 1979. He is a senior researcher at the Process Recognition Department of the Institute of Mathematics and Informatics and an associated professor at the Gediminas Technical University. His research interest include the classical and robust approaches of dynamic system identification as well technological process control. 


\section{Apie Hameršteino sistemu, turinčiu dalimis tiesiška netiesiškumą su teigiamais nuožulnumais, identifikavima}

\section{Rimantas PUPEIKIS}

Straipsnyje nagrinėjamas Hameršteino sistemų laipsniškas dalimis tiesiško netiesiškumo su nežinomais nuožulnumais bei nežinomu slenksčiu ir tiesinès dalies, aprašomos skirtumine lygtimi su nežinomais koeficientais, junginys. Parodyta, kad pertvarkius ięjimo signalo stebẻjimus pagal didejjančias jų reikšmes, galima išskirti vidurinę stebèjimų dali, atitinkančią nestebimo tarpinio signalo stebejjimus. Pasiūlytas pilno tarpinio signalo atstatymo būdas pagal ièjimo signalo vidurinès dalies ir atitinkamus išẻjimo signalo stebejjimus. Nežinomų tiesinès Hameršteino sistemos dalies koeficientu ir dalimis tiesiško netiesiškumo parametrų bei slenksčiu įverčiai gaunami mažiausiuju kvadratų metodo algoritmais, apdorojant stebimų pertvarkytu ięjimo, išèjimo bei atkurto tarpinio signalu duomenis. Pateikti modeliavimo rezultatai. 